\title{
Denosumab-induced hypocalcaemia in metastatic castrate-resistant prostate cancer
}

\author{
Florence Gunawan, Elizabeth George and Mark Kotowicz
}

\section{Summary}

Denosumab is a fully human MAB that acts as a potent anti-resorptive by inhibiting activation of osteoclasts by inhibiting the receptor activator of nuclear factor-kappa B (RANK) ligand. Hypocalcaemia has been reported as one of the serious adverse sequelae of use of denosumab. We present a case of refractory hypocalcaemia following administration of a single dose of denosumab in a patient with metastatic castrate-resistant prostate cancer. The patient's serum calcium and vitamin D concentrations and renal function were normal prior to denosumab administration. Serum alkaline phosphatase (ALP) level was however elevated pre-morbidly consistent with known bone metastases. The patient was treated with high-dose oral and IV calcium without any appreciable response in serum calcium. During his 30-day hospital admission, he demonstrated disease progression with development of new liver metastases and bone marrow involvement. Normocalcaemia was not achieved despite 1 month of aggressive therapy. Given the patient was asymptomatic and prognosis guarded, he was eventually discharged for ongoing supportive care under the palliative care team.

\section{Learning points:}

- Denosumab is a potent anti-resorptive therapy and hypocalcaemia is one of the known adverse effects.

- Serum calcium and vitamin D concentrations must be replete prior to administration of denosumab to reduce the risk of hypocalcaemia.

- Denosumab has been proven to be more effective than zoledronic acid in preventing skeletal-related adverse effects in patients with metastatic castrate-resistant prostate cancer.

\section{Background}

Denosumab is a potent anti-resorptive and its use has been proven to be effective in preventing skeletal-related adverse effects in patients with metastatic castrateresistant prostate cancer. Hypocalcaemia has been reported as one of the serious side effects of denosumab. Although often mild and transient, hypocalcaemia can lead to significant morbidity or even mortality. To our knowledge, this is the first case report of a patient with metastatic castrate-resistant prostate cancer who received a single dose of denosumab and developed profound refractory hypocalcaemia in the absence of preceding anti-resorptive use and other predisposing risk factors.

\section{Case presentation}

A 65-year-old man with castrate-resistant adenocarcinoma of the prostate with bone metastases presented with hypocalcaemia following a single dose of denosumab. He was diagnosed in 2015 and had previously received five cycles of docetaxel chemotherapy. Therapy was 
ceased prematurely due to complication of severe febrile neutropaenia. He was continued on androgen deprivation therapies including Degarelix, Bicalutamide and Goserelin. He received ten fractions of palliative radiotherapy to the lumbosacral spine for management of pain from bone metastasis. Denosumab was commenced as an adjunct therapy for management of bone metastatic disease. He did not have preceding antiresorptive use.

Prior to the diagnosis of the prostate cancer, he was well and took no regular medications. His family history was significant for prostate cancer affecting his father who deceased aged 80 years.

Prior to denosumab, his serum biochemistry was normal with a creatinine of $75 \mu \mathrm{mol} / \mathrm{L}(30-90 \mu \mathrm{mol} / \mathrm{L})$, eGFR $>90 \mathrm{ml} / \mathrm{min} / 1.73 \mathrm{~m}^{2}$, corrected calcium of 2.43 $\mathrm{mmol} / \mathrm{L}$ (2.15-2.55 mmol/L), albumin $38 \mathrm{~g} / \mathrm{L}$ (34-45), phosphate $1.28 \mathrm{mmol} / \mathrm{L}(0.8-1.5 \mathrm{mmol} / \mathrm{L})$ and 25-hydroxy vitamin D of $102 \mathrm{nmol} / \mathrm{L}(50-250 \mathrm{nmol} / \mathrm{L})$. Alkaline phosphatase (ALP) was elevated at $790 \mathrm{U} / \mathrm{L}$ (35-110 U/L), consistent with the known osteoblastic bone metastases. He was commenced on cholecalciferol 1000 IU daily and calcium carbonate $600 \mathrm{mg}$ daily and administered a single s.c. dose of $120 \mathrm{mg}$ of denosumab.

Seven days later, routine outpatient biochemistry revealed new hypocalcaemia with a corrected calcium of $1.93 \mathrm{mmol} / \mathrm{L}$ (2.15-2.55 mmol/L), albumin $37 \mathrm{~g} / \mathrm{L} \mathrm{(34-45).}$ He was initially asymptomatic and managed with up-titration of calcium carbonate to $3600 \mathrm{mg}$ daily. Three

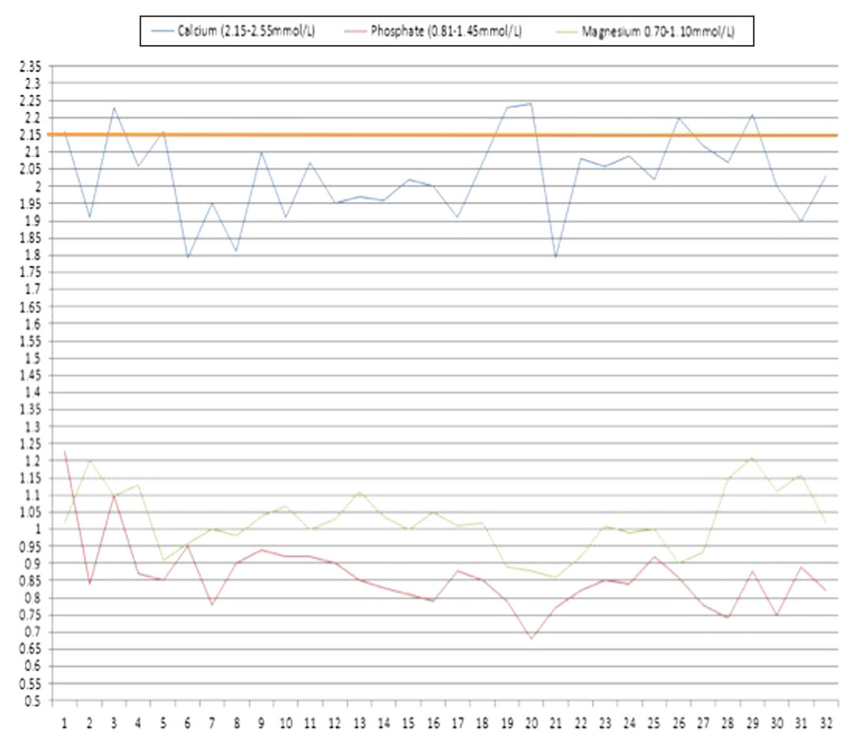

Figure 1

Graph demonstrating fluctuations in the serum calcium levels despite intravenous and oral replacement. The values almost exclusively fall below the reference range marked by the orange line. weeks later, he became symptomatic with lethargy, perioral numbness, fingertips paresthesia and leg cramping. Repeat corrected calcium was further reduced to 1.91 $\mathrm{mmol} / \mathrm{L}$ with an appropriately elevated parathyroid (PTH) hormone concentration of $30.1 \mathrm{pmol} / \mathrm{L}$ (2.0-9.3 pmol/L), albumin $36 \mathrm{~g} / \mathrm{L}$ (34-50), low phosphate of $0.84 \mathrm{mmol} / \mathrm{L}$ (0.8-1.4 mmol/L), Mg $1.2 \mathrm{mmol} / \mathrm{L}$ (0.70-1.05 mmol/L), 25-hydroxy vitamin D $77 \mathrm{nmol} / \mathrm{L}$ and ALP 625 U/L (35110 U/L). Chvostek's and Trousseau's signs were negative and the QTC interval on the ECG was normal at $449 \mathrm{~ms}$. He was admitted to hospital for further management.

During admission, large doses of continuous i.v. 10\% calcium gluconate $(2.2 \mathrm{mmol} / 1 \mathrm{~g} / 10 \mathrm{ml})$, up to $44 \mathrm{mmol} /$ day were required to maintain his serum calcium at the lower limit of normal. Calcium carbonate was switched to calcium citrate $1000 \mathrm{mg}$ QID to improve absorption given his concomitant proton pump inhibitor use. Cholecalciferol was increased to 1000 IU BD. He was also commenced on calcitriol with the dose titrated up to $6 \mu \mathrm{g}$ daily and hydrochlorothiazide $25 \mathrm{mg}$ daily in an attempt to reduce renal tubular excretion of calcium. Despite this aggressive therapy, the hypocalcaemia remained refractory (Fig. 1). Oral dexamethasone $4 \mathrm{mg} \mathrm{BD}$ was trialed in an attempt to induce bone resorption.

Bone turnover markers were elevated with a fasting C-telopeptide (CTX) of 696 ng/L (100-600 ng/L) and Procollagen 1 (P1NP) of $256 \mu \mathrm{g} / \mathrm{L}(15-80 \mu \mathrm{g} / \mathrm{L})$. Urine calcium was low at $<1.3 \mathrm{mmol} / \mathrm{L}$; hence, a 24 -h urine calcium excretion was not able to be accurately calculated. Renal function remained stable during hospital stay.

Despite a month long of ongoing high-dose i.v. and oral calcium therapy, the highest corrected calcium achieved was $2.23 \mathrm{mmol} / \mathrm{L}$, albumin $29 \mathrm{~g} / \mathrm{L}$ (34-50). Intravenous calcium was ceased for 2 days on day 30; however, this was recommenced as serum calcium dropped to $1.90 \mathrm{mmol} / \mathrm{L}$, albumin $31 \mathrm{~g} / \mathrm{L}$ (34-50). At the same time, there was evidence of ongoing disease progression with markedly deranged liver function tests and pancytopaenia, suggestive of bone marrow disease. A bone scan demonstrated progressive skeletal involvement (Fig. 2). He was deemed unsuitable for further chemotherapy trials and was subsequently referred to the palliative care team. He was discharged home on day 33 on oral calcium replacement.

\section{Outcome and follow-up}

He was discharged home under the palliative care team on oral calcium replacement. 


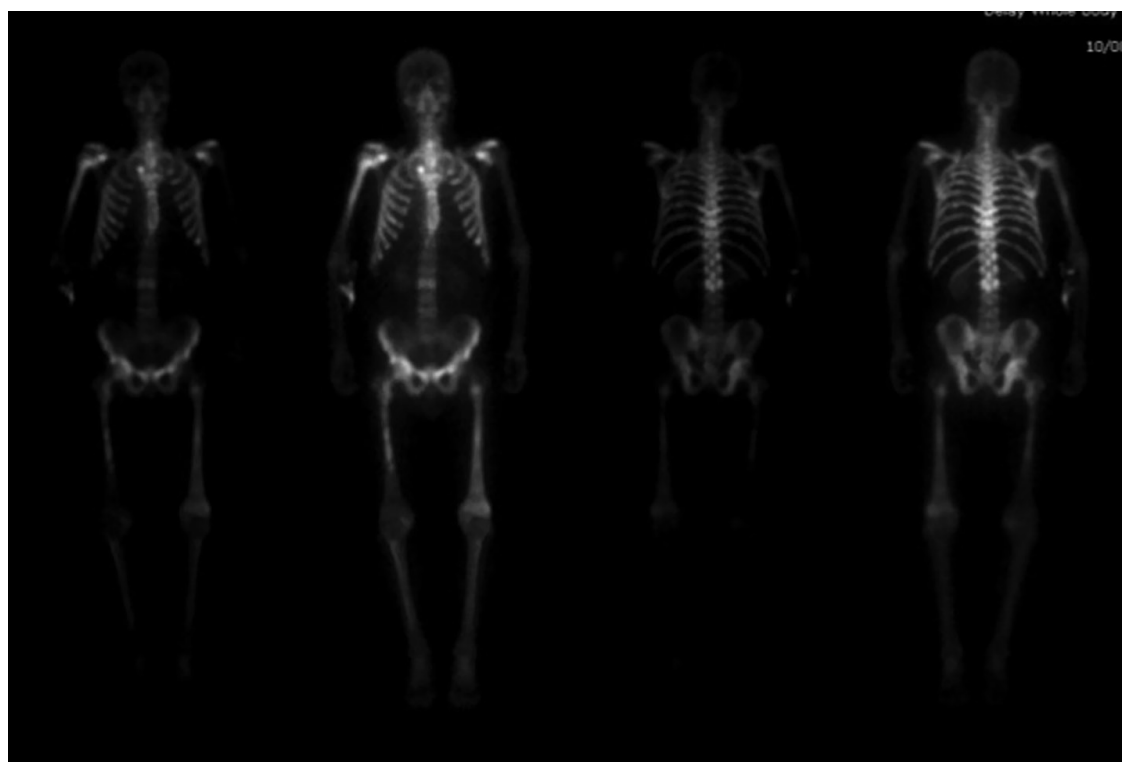

\section{Figure 2}

Whole-body bone scan showing abnormal focal and confluent skeletal activity in the left calvarium, segments of cervical, thoracic and lumbosacral spine, bilateral pelvis, bilateral ribs, sternum, scapulae, right humerus and bilateral femoral shafts consistent with metastatic bone disease.

\section{Discussion}

Prostate cancer is the leading malignancy in the aging male population. Bone metastases represent a major disease burden in men with advanced prostate cancer. The axial skeleton is predominantly affected and lesions are usually osteoblastic/sclerotic in nature, although lytic lesions have also been reported. The mechanism by which prostate cancer induces osteoblastic or osteoclastic changes remain poorly understood. Patients with bone metastases are often initially asymptomatic before insidious development of pain. This may be more abrupt in the setting of a pathological fracture and may lead to complications such as nerve root entrapment or spinal cord compression.

Regular bisphosphonate and denosumab use have been shown to decrease the incidence of skeletalrelated events (SRAEs) in patients with metastatic hormone-resistant prostate cancer $(1,2)$. The use of bisphosphonates in metastatic hormone-sensitive prostate cancer does not significantly decrease the rate of SRAE (3) and there are no data on denosumab for this.

In the hormone-resistant prostate cancer, denosumab has been proven to be more effective than zoledronic acid in preventing SRAE. Hypocalcaemia with denosumab, however, occurs significantly more frequently, consistent with its greater anti-resorptive effect (2). Although often mild and transient, hypocalcaemia can lead to significant morbidity and in some cases mortality.

The Food and Drug Administration (FDA) and Therapeutic Goods Administration (TGA) have approved the use of denosumab $120 \mathrm{mg}$ four weekly for the prevention of skeletal-related events in patients with bone metastases from solid tumours. This dose is significantly higher than the usual dose for management of osteoporosis (60 mg every 6 months). The inherent risk for hypocalcaemia due to osteoblastic bone metastases and the potent anti-resorptive effect of denosumab render this population particularly at high risk for developing hypocalcaemia (4).

Other risk factors, which may potentiate the hypocalcaemia risk, include renal impairment, vitamin D deficiency and gastrointestinal calcium loss from nausea, vomiting or diarrhoea. An open-label pharmacokinetics and safety study of a single $60 \mathrm{mg}$ s.c. dose of denosumab found an incidence of hypocalcaemia in $18.6 \%$ of subjects with varying degree of renal impairment, defined as a creatinine clearance of $<60 \mathrm{~mL} / \mathrm{min}$ by CockcroftGault estimation (5). Chemotherapy agents, such as cisplatin, can also cause hypomagnesemia with secondary hypocalcaemia due to reduced parathyroid hormone (PTH) production, which may further contribute to hypocalcaemia.

In the present case, the profound hypocalcaemia occurred in the absence of the above risk factors. It developed 1 week following denosumab and was refractory to treatment despite more than 30 days of intensive therapy.

High-grade hypocalcaemia has been reported in the literature of men with metastatic prostate cancer who received at least one dose of $120 \mathrm{mg}$ of denosumab (6). In a study of 42 men, severe hypocalcaemia developed after the first dose in seven patients, with a median time from drug administration to calcium nadir of 25 days (14-106 days) 
and a median recovery time to baseline calcium was 17 days (range: 6-40 days) (6).

Our patient received a total of $187 \mathrm{~g}$ of i.v. calcium, $128 \mathrm{~g}$ of elemental oral calcium and high-dose oral calcitriol replacement. Despite this, there was ongoing difficulty in maintaining normocalcaemia. Adnan et al. reported a case of a 45-year-old man with castrate-resistant metastatic prostate cancer with three previous zoledronic acid infusions before switching to denosumab, who presented with profound hypocalcaemia with corrected calcium of $1.12 \mathrm{mmol} / \mathrm{L}$, in the setting of vitamin $\mathrm{D}$ deficiency. He required $80 \mathrm{~g}$ of i.v. calcium over a 2-week period along with high calcium bath haemodialysis (7). Laskowski et al. reported a 66-year-old man with metastatic prostate and small-cell lung cancer with hypocalcaemia and hypomagnesaemia 2 weeks following first dose of denosumab administration and concomitant chemotherapy with cisplatin. This patient received $16 \mathrm{~g}$ of i.v. calcium along with oral supplementation and was discharged on day 4 (8).

To our knowledge, this is the first case report of a patient with metastatic castrate-resistant prostate cancer who received a single dose of denosumab and developed refractory hypocalcaemia in the absence of other predisposing risk factors. His case also represents the most severe to date in the literature requiring prolonged hospital admission for management of the denosumabinduced hypocalcaemia.

Given the risk of hypocalcaemia, clinicians should ensure that serum calcium is normal and vitamin D levels are replete prior to denosumab administration. Renal function and pre-existing electrolyte disturbance should also be corrected to identify patients at risk.

The serum ALP represents an adjunct measure, which may also be used to predict those who are at risk of developing hypocalcaemia. A study of 219 patients with metastatic bone disease from solid tumours reported grade 2-3 hypocalcemia in 10.4\% from 368 denosumab administrations. Patients with higher ALP were at higher risk for hypocalcaemia and the cut-off value to predict denosumab-related hypocalcaemia was $587 \mathrm{U} / \mathrm{L}$ with a sensitivity of 0.77 and specificity of 0.81 (9).

Once treatment has started, close monitoring of serum calcium should be undertaken, especially in the first weeks of initiating therapy. Currently, the denosumab product information (PI) statement advises to administer calcium and vitamin $\mathrm{D}$ as necessary to treat or prevent hypocalcaemia. There remains, however, a lack of recommendation to check vitamin $\mathrm{D}$ levels and the 'adequate' levels for which denosumab administration is safe. The PI also warns that patients with severe renal impairment $(\mathrm{CrCl}<30 \mathrm{~mL} / \mathrm{min})$ are at risk of developing hypocalcaemia; however, no dose adjustments are recommended.

Given the sequelae of complications of hypocalcaemia, clinicians are advised to remain vigilant in assessing and monitoring patients following administration of denosumab, particularly those patients who have predisposing risk factors to developing this.

\section{Declaration of interest}

The authors declare that there is no conflict of interest that could be perceived as prejudicing the impartiality of this case report.

\section{Funding}

This research did not receive any specific grant from any funding agency in the public, commercial or not-for-profit sector.

\section{Patient consent}

Patient is deceased and consent could not be obtained from family members.

\section{Author contribution statement}

F G consolidated information including investigation results and treatment and helped write up case report. E G consolidated information including investigation results and treatment, and write up case report. M K supervised the case report.

\section{References}

1 Himelstein AL, Foster JC, Khatcheressian JL, Roberts JD, Seisler DK, Novotny PJ, Qin R, Go RS, Grubbs SS, O'Connor T, et al. Effect of longer-interval vs standard dosing of zoledronic acid on skeletal events in patients with bone metastases: a randomized clinical trial. JAMA 2017317 48-58. (https://doi.org/10.1001/jama.2016.19425)

2 Fizazi K, Carducci M, Smith M, Damião R, Brown J, Karsh L, Milecki P, Shore N, Rader M, Wang H, et al. Denosumab versus zoledronic acid for treatment of bone metastases in men with castration-resistant prostate cancer: a randomised, double-blind study. Lancet 2011377 813-822. (https://doi.org/10.1016/S0140-6736(10)62344-6)

3 Smith MR, Halabi S, Ryan CJ, Hussain A, Vogelzang N, Stadler W, Hauke RJ, Monk JP, Saylor P, Bhoopalam N, et al. Randomized controlled trial of early zoledronic acid in men with castrationsensitive prostate cancer and bone metastases: results of CALGB 90202 (alliance). Journal of Clinical Oncology 201432 1143-1150. (https://doi.org/10.1200/JCO.2013.51.6500)

4 Murray RM, Grill V, Crinis N, Ho PW, Davison J \& Pitt P. Hypocalcemic and normocalcemic hyperparathyroidism in patients with advanced prostatic cancer. Journal of Clinical Endocrinology and Metabolism 2001 86 4133-4138. (https://doi.org/10.1210/jcem.86.9.7864)

5 Block GA, Bone HG, Fang L, Lee E \& Padhi D. A single-dose study of denosumab in patients with various degree of renal impairment. Journal of Bone and Mineral Research 201227 1471-1479. (https://doi. org/10.1002/jbmr.1613) 
6 Autio KA, Farooki A, Glezerman IG, Chan A, Schneider CW, Barr HC, Seyboth BM, Kampel LJ, Danila DC, Rathkopf DE, et al. Severe hypocalcemia associated with denosumab in metastatic castrationresistant prostate cancer: risk factors and precautions for treating physicians. Clinical Genitourinary Cancer 201513 e305-e309. (https:// doi.org/10.1016/j.clgc.2014.11.008)

7 Adnan MM, Bhutta U, Iqbal T, AbdulMujeeb S, Haragsim L \& Amer S. Severe hypocalcemia due to denosumab in metastatic prostate cancer. Case Reports in Nephrology 20142014565393.
8 Laskowski LK, Goldfarb DS, Howland MA, Kavcsak K, Lugassy DM \& Smith SW. A RANKL wrinkle: denosumab-induced hypocalcemia. Journal of Medical Toxicology 201612 305-308. (https://doi org/10.1007/s13181-016-0543-y)

9 Kinoshita Y, Arai M, Ito N, Takashi Y, Makita N, Nangaku M, Shinoda Y \& Fukomoto S. High serum ALP level is associated with increased risk of denosumab-related hypocalcaemia in patients with bone metastases from solid tumours. Endocrine Journal $2016 \mathbf{6 3}$ 479-484. (https://doi.org/10.1507/endocrj.EJ16-0003)

Received in final form 6 October 2019

Accepted 25 October 2019 